\title{
Assessment of interleukin-17A, C5a and RANTES for early diagnosis of neonatal sepsis - a preliminary study
}

\author{
BEATA KASZTELEWICZI, EWA PIOTROWSKA², JUSTYNA TOEŁOCZKO , \\ MARIA K. BORSZEWSKA-KORNACKA ${ }^{3}$, HANNA GREGOREK ${ }^{1}$, \\ KATARZYNA DZIERŻANOWSKA-FANGRAT ${ }^{l}$ \\ ${ }^{1}$ Department of Clinical Microbiology and Immunology, The Children's Memorial Health Institute, Warsaw, Poland \\ ${ }^{2}$ Neonatal Intensive Care Unit, The Nicolaus Copernicus Hospital in Olsztyn, Olsztyn, Poland \\ ${ }^{3}$ Department of Neonatology and Neonatal Intensive Care, Medical University of Warsaw, Warsaw, Poland
}

\begin{abstract}
The aim of the present study was to investigate serum levels of novel markers: interleukin 17A (IL17A), anaphylatoxin C5a and chemokine regulated upon activation normal T-cell expressed and secreted (RANTES) in neonates with clinically suspected early-onset neonatal sepsis (EONS), and to compare their values with those of non-infected neonates. Eighteen neonates with clinical signs and symptoms of EONS were enrolled in this study. Fifty healthy, non-infected neonates served as the control group. In all neonates serum levels of IL-17A, C5a and RANTES were measured by solid-phase sandwich enzyme-linked immunosorbent assay (ELISA). At the time of investigation serum levels of anaphylatoxin C5a were significantly higher in neonates with clinical symptoms of EONS than in non-infected neonates (median 65.35 vs. $50.4 \mathrm{ng} / \mathrm{ml}, \mathrm{p}=0.034$ ), whereas levels of RANTES were similar and levels of IL-17A were under detection limit of the method. Based on these preliminary results, serum levels of C5a may be a useful marker of inflammation in early onset neonatal sepsis. Because traditional methods of microbiological diagnostics in EONS are frequently unsuccessful, the search for an alternative laboratory biomarkers is of great clinical importance. Thus, there is a strong need for further studies evaluating usefulness of this anaphylatoxin in EONS diagnosis on a larger group of patients.
\end{abstract}

Key words: RANTES, early-onset neonatal sepsis, anaphylatoxin C5a.

(Cent Eur J Immunol 2016; 41 (4): 376-382)

\section{Introduction}

Early-onset neonatal sepsis (EONS), defined as occurring in the first 3 days of life, continues to be a leading cause of morbidity and mortality in neonates [1]. Diagnosis of sepsis is a formidable challenge for neonatal intensive care units (NICU) because clinical signs of sepsis are nonspecific, subtle and are observed in other non-infectious conditions. Furthermore, bacterial cultures are time-consuming, frequently false negative, especially in neonates, due to low or intermittent bacteraemia, small blood inoculation volumes or intrapartum antibiotics use [2]. Recently performed meta-analysis of molecular assays used for early detection of bacteraemia in neonates have yielded disappointing results compared with blood cultures [3]. Thus, despite its limitations, blood cultures still remain a 'gold standard'. To assist in the diagnosis of neonates with suspected sepsis, many laboratory tests including whole blood cell count, differential, immature-to-total neutrophils (I/T) ratio, along with acute-phase reactants such as C-reactive protein (CRP) and procalcitonin (PCT), are routinely used [1]. However, so far none of the single laboratory tests can provide reliable and early identification of neonates with sepsis. This lead to search for more informative markers (or combinations thereof). The recent interest has focused on chemokines, cytokines and acute phase reactants (and other substances associated with the inflammatory response), whose serum levels may reflect the host's immunological status and response to infection [4-6].

Interleukin 17A (IL-17A) is a cytokine secreted mainly by a recently characterized distinct subset of helper T cells - Th17 cells. The Th17 cells are considered to be important for immunity to microorganisms that are not eliminated by Th1 or Th2-cell mediated response [7]. Interleukin 17A mediates pro-inflammatory response by triggering the production of many other cytokines, such as IL-1 $\beta$, IL-6 and

Correspondence: Beata Kasztelewicz, Department of Clinical Microbiology and Immunology, The Children's Memorial Health Institute,

Warsaw, Poland, e-mail: b.kasztelewicz@czd.pl

Submitted: 5.02.2016, Accepted: 26.04.2016 
tumor necrosis factor (TNF) and orchestrates the response of both innate and adaptive immune cells [8]. Elevated levels of IL-17A were found in the experimental model of sepsis [9]. Neutralization of IL-17A exerted a protective effect on sepsis mortality, characterized by decreased bacteraemia and a decreased level of inflammatory cytokines [9]. Studies conducted recently in an adult population revealed a significantly higher IL-17 production in patients with severe sepsis than in healthy controls $[10,11]$. Data for neonatal populations are limited $[12,13]$.

$\mathrm{C} 5 \mathrm{a}$ is considered a central mediator in the immunopathogenesis of sepsis, as it modulates other systems, including the coagulation cascade and the release of proinflammatory cytokines [14]. Large amounts of anaphylatoxin $\mathrm{C} 5 \mathrm{a}$ are generated during the early stages of sepsis [15]. Excessive C5a production, in turn, elicits numerous harmful effects $[7,15]$. On the other hand, it has been demonstrated that blockage of C5a greatly improve the survival of rodents during sepsis [16]. Significantly enhanced levels of C5a have been found in septic shock in adult patients compared with healthy individuals [17], but none of the study has evaluated this anaphylatoxin in the setting of neonatal sepsis, so far.

Regulated on activation, normal $\mathrm{T}$ cell expressed and secreted (RANTES), also known as chemokine (C-C motif) ligand 5 (CCL5), mediates the trafficking and homing of T helper cells, monocytes and eosinophils into inflammatory sites. An inverse correlation has been reported between the levels of RANTES and disease severity, and mortality in patients with sepsis and lower levels of this chemokine were found in non-surviving patients, suggesting its beneficial pro-inflammatory role in host response to infection [18]. A few studies reported RANTES levels in infected neonates [19-22] including those with EONS $[20,22]$. Most of them documented lower RANTES levels in infected neonates compared to non-infected controls. However, contradictory observation has also been reported [23].

The aim of this preliminary study was to investigate serum levels of IL-17A, anaphylatoxin C5a and chemokine RANTES in neonates with EONS and those non-infected, and to evaluate the potential utility of these novel markers for early diagnosis of neonatal sepsis.

\section{Material and methods}

\section{Subjects}

A total of 68 neonates were enrolled prospectively in the Neonatal Intensive Care Unit (NICU) at The Nicolaus Copernicus Hospital in Olsztyn and in the tertiary NICU at the Medical University of Warsaw.

Neonates eligible for inclusion in the case group had to have clinical signs of early-onset neonatal sepsis (EONS) developed within 72 hours of life and/or history of factors associated with increased risk for infection.

Clinical signs of sepsis were defined as the presence of three or more of the following clinical manifestations, without any other obvious pathological cause: temperature instability (core temperature $\geq 38.5$ or $\leq 36^{\circ} \mathrm{C}$ ); respiratory symptoms (apnoea, tachypnoea with respiratory rate $>60$ per minute, cyanosis, need for high ventilator settings or oxygen); cardiovascular symptoms including hypotension (blood pressure $<5^{\text {th }}$ percentile for age), tachycardia (heart rate $>160$ per minute), bradycardia (heart rate $<80$ per minute), or poor perfusion; neurological symptoms (hypotonia, hyporeflexia, irritability, lethargy and seizures); gastrointestinal symptoms (poor feeding, abdominal distension, green or bloody residuals, vomiting).

Risk factors were: maternal chorioamnionitis, mothers with inadequately treated group B streptococcus (GBS) colonisation, mothers non-tested for GBS with premature rupture of membranes (PROM) for $>18$ hours, maternal fever during labour or premature labour [1].

Newborns with the above-mentioned clinical findings and/or risk factors have had sepsis screening laboratory tests, including: acute phase reactant $-\mathrm{C}$-reactive protein (CRP concentrations $>5 \mathrm{mg} / \mathrm{l}$ were considered abnormal), white blood cell (WBC) differential count (WBC $>35 \times$ $10^{9} / 1$ or $<5 \times 10^{9} / 1$ was considered abnormal) and immature to total neutrophil ratio (I/T ratio $>0.2$ was considered abnormal), and platelet counts (PLT $<80 \times 10^{9} / 1$ was considered abnormal).WBC, neutrophil and immature WBC counts were measured using an Sysmex XT-1800i Automated Hematology Analyzer (Sysmex America, Mundelein, USA). The plasma levels of CRP were determined using Roche Cobas Integra 800 full-automated analyzer (Roche Diagnostics, Mannheim, Germany). At the same time, an additional blood sample was provided for quantitative analysis of IL-17A, C5a and RANTES. Blood cultures were performed on every patient with suspected infection.

Inclusion criteria for the case group were the presence of clinical signs of sepsis and/or the presence of risk factors plus at least two abnormal laboratory test results. Diagnosis of respiratory distress syndrome, necrotizing enterocolitis, meconium aspiration syndrome, hypoxia, intracranial haemorrhage or congenital heart disease was ruled-out in all neonates included in the study.

The variables of each case recorded included newborn's demographics, prenatal and postnatal history, physical examination findings, vital signs, routine blood test, microbiologic culture results and applied antibiotic therapy.

The criteria for inclusion to the control group were neither evidence of infection in neonate nor perinatal risk factors and a whole blood sample collected within the first 72 hours of life available for assessment of IL-17A, C5a and RANTES. Data recorded for healthy neonates includ- 
ed: gestational age, gender, delivery mode, birth weight, five-minute Apgar score and CRP level.

The study was conducted in accordance with the Declaration of Helsinki and written informed consent was obtained from the parents or legal guardians. The study protocol was approved by the Ethical Committee of the Children's Memorial Health Institute.

\section{Measurement of IL-17A, C5a and RANTES by ELISA}

For the IL-17A, C5a and RANTES analysis $1 \mathrm{ml}$ of the venous blood was collected into test tubes without anticoagulants and allowed to clot for 30 minutes and then centrifuged at $4^{\circ} \mathrm{C}$ for 10 minutes at $1000 \times \mathrm{g}$. Each neonate's serum sample was separated into 3 aliquots and stored immediately at $-20^{\circ} \mathrm{C}$ until analysis.

Interleukin 17A, C5a and RANTES were measured using commercially available solid-phase sandwich enzyme-linked immunosorbent assay (ELISA) kits (Human IL-17A ELISA development HRP kit, MABTECH AB, Nacka Strand, Sweden for IL-17A; BD OptEIA Human C5a ELISA Kit II, Becton, Dickinson and Company BD Biosciences, San Jose, CA, USA for C5a; Human RANTES ELISA Construction Kit, Antigenix America Inc., Melville, NY, USA for RANTES). The detection limit was $4 \mathrm{pg} / \mathrm{ml}, 0.047 \mathrm{ng} / \mathrm{ml}$ and $0.016 \mathrm{ng} / \mathrm{ml}$ for IL-17A, C5a and RANTES, respectively.

All clinical specimens were transported frozen on dry ice and tested simultaneously to ensure the uniformity of measurements. No sample was thawed until the analysis. Assays for IL-17A, C5a and RANTES were performed in the Department of Clinical Microbiology and Immunology, the Children's Memorial Health Institute in Warsaw.

\section{Statistical analysis}

Categorical variables were expressed as number and percentage. Normally distributed continuous variables were summarized as mean \pm standard deviation (SD), whereas other continuous variables were presented as median and $25^{\text {th }}$ to $75^{\text {th }}$ percentiles (interquartile range, IQR). Statistical significances of the differences in serum C5a and RANTES levels between different study groups were assessed by the Mann-Whitney $U$ test. For correlation analysis, Spearman's correlation coefficients were calculated. Statistical analysis was done using Statistica software version 6.0 (StatSoft. Inc, Tulsa, USA). A $p$-value of $<0.05$ was considered significant.

\section{Results}

A total of 68 neonates were prospectively enrolled in this preliminary study within the first 72 hours after birth. Eighteen neonates with clinical signs and symptoms of systemic infection formed the EONS group, whereas 50 neonates without evidence of infection were classified into the control group. As compared with non-infected neonates, newborns with EONS were more likely to have a lower gestational age. The groups differed also in delivery mode and, as expected,in CRP concentration (Table 1). There was no difference according to birth weight, length or male/female ratio.

Regarding neonates in the EONS group, the diagnosis was based on clinical grounds supported by laboratory tests. No patients had a positive blood culture. At the time of the sepsis episode 9 neonates presented respiratory distress and 6 of them had a diagnosis of pneumonia. Detailed clinical characteristics of the EONS group were summarized in Table 2.

At the time of sepsis investigation serum levels of C5a in the EONS group were significantly higher compared to those in the non-infected group (median 65.35 vs. $50.4 \mathrm{ng}$ / $\mathrm{ml}, p=0.034$; Fig. 1A), whereas no significant difference in RANTES levels between the two groups were found (median 188.95 vs. $195.2 \mathrm{ng} / \mathrm{ml} ; p=0.444$; Fig. 1B).

Levels of IL-17A were under the detection limit $(<4$ $\mathrm{pg} / \mathrm{ml}$ ) in both EONS and non-infected neonates. IL-17A was excluded from further analysis because of having val-

Table 1. Demographic and clinical characteristics of septic neonates (EONS group, $n=18$ ) and non-infected neonates (control group, $n=50$ )

\begin{tabular}{|c|c|c|c|}
\hline Characteristics & EONS $(n=18)$ & Non-infected controls $(n=50)$ & $P$-values ${ }^{\text {a }}$ \\
\hline Male gender, $n(\%)$ & $10(55.6)$ & $24(48.0)$ & 0.784 \\
\hline Gestational age, Me (IQR) & $38.5(37-39)$ & $40(39-40)$ & 0.003 \\
\hline Length, mean $\pm \mathrm{SD}$, cm & $53.94 \pm 4.16$ & $55.04 \pm 3.15$ & 0.257 \\
\hline Birth weight, mean $\pm \mathrm{SD}, \mathrm{g}$ & $3296.67 \pm 691.46$ & $3555.20 \pm 503.19$ & 0.096 \\
\hline Vaginal delivery, $n(\%)$ & $7(38.9)$ & $38(76.0)$ & 0.008 \\
\hline Apgar score at $5 \mathrm{~min}, \mathrm{Me}(\mathrm{IQR})$ & $10(9-10)$ & $10(10-10)$ & 0.118 \\
\hline CRP level $^{\mathrm{b}}, \mathrm{Me}$ (IQR), ng/ml & $41.4(26.5-84.00)$ & $1.8(1.2-3.60)$ & 0.000003 \\
\hline
\end{tabular}

Me - median; IRQ - interquartile range; EONS - early-onset neonatal sepsis; $C R P-C$-reactive protein

${ }^{a} P$-values by Student t test, Mann-Whitney U test or Fisher's exact test as appropriate; bold indicated statistical differences.

${ }^{b}$ Measured between $23^{\text {th }}$ and $25^{\text {th }}$ hour of life. 
Table 2. Detailed characteristics of neonates with EONS

\begin{tabular}{|c|c|}
\hline Characteristics & $n=18$ \\
\hline Preterm $(<37 \mathrm{wk})$ & $2(11.1)$ \\
\hline Small for gestational age ${ }^{a}$ & $2(11.1)$ \\
\hline Positive blood culture & 0 \\
\hline Positive culture of other material ${ }^{\mathrm{b}}$ & $3(16.7)$ \\
\hline Haemoglobin, mean $\pm \mathrm{SD}, \mathrm{g} / \mathrm{dl}$ & $17.33 \pm 2.01$ \\
\hline White blood cell count, mean $\pm \mathrm{SD}, \times 10^{9} / 1$ & $22.25 \pm 12.13$ \\
\hline Abnormal white blood cell count $\left(>35 \times 10^{9} / 1\right.$ or $\left.<5 \times 10^{9} / 1\right)$ & $2(11.1)$ \\
\hline $\mathrm{I} / \mathrm{T}$ ratio $(>0.2)$ & $5(27.8)$ \\
\hline Platelet count, mean $\pm \mathrm{SD}, 10^{9} / 1$ & $233.75 \pm 77.64$ \\
\hline Thrombocytopenia $\left(<80 \times 10^{9} / 1\right), n(\%)$ & 0 \\
\hline Temperature instability (hypothermia $\leq 36^{\circ} \mathrm{C}$ or fever $>38^{\circ} \mathrm{C}$ ) & 0 \\
\hline Infection source ${ }^{c}$ & $6(33.4)$ \\
\hline Time of symptoms onset (hours after birth), Me (IQR) & $2(1.0-16.5)$ \\
\hline Respiratory symptoms: apnoea, cyanosis, tachypnoea with respiratory rate $>60$ per minute, oxygen dependence & $9(50.0)$ \\
\hline $\begin{array}{l}\text { Cardiovascular symptoms: hypotension with blood pressure }<5^{\text {th }} \text { percentile for age, tachycardia with heart rate } \\
>160 \text { per minute, bradycardia with heart rate }<80 \text { per minute, poor perfusion }\end{array}$ & $4(22.2)$ \\
\hline Neurological symptoms: hypotonia, hyporeflexia, irritability, lethargy and seizures & $2(11.1)$ \\
\hline Gastrointestinal symptoms: poor feeding, abdominal distension, green or bloody residuals, vomiting & 0 \\
\hline Chorioamnionitis $^{\mathrm{d}}$ & $7(38.9)$ \\
\hline Maternal colonization with GBS & $10(55.6)$ \\
\hline Intrapartum prophylaxis $>4$ hours & $1(5.6)$ \\
\hline PROM (> 18 hours) & $1(5.6)$ \\
\hline
\end{tabular}

I/T ratio-immature to total neutrophil count; Me - median; IQR-interquartile range; GBS - Group B Streptococcus; PROM - premature rupture of membranes. Note: data in parenthesis are percentages.

${ }^{a}$ Birth size assessed according to Olsen et al. [30].

${ }^{b}$ E. coli, S. viridans, cultured from ear swabs and throat, respectively.

${ }^{c}$ Diagnosis of pneumonia in all cases.

${ }^{d}$ Defined as maternal fever $\left(>38^{\circ} \mathrm{C}\right)$, and at least two of the following criteria: maternal leukocytosis $\left(\right.$ WBC $\left.>15 \times 10^{\circ} /\right)$, maternal tachycardia $($ heart rate $>100$ per minute), foetal tachycardia (heart rate $>160$ per minute), uterine tenderness and/or foul odour of the amniotic fluid.

ues below the assay working range, which would not enable an examination as a continuous variable.

A separate analysis was performed to determine whether there was a difference between premature and term neonates as well as among neonates according to delivery mode. Concentrations of C5a and RANTES did not differ between premature and term neonates (median C5a level 46.1 vs. $52.7 \mathrm{ng} / \mathrm{ml} ; p=0.397$ and median RANTES level 254.1 vs. $185.7 \mathrm{ng} / \mathrm{ml} ; p=0.367$, for premature and term neonates, respectively). Similarly, no significant difference was observed according to delivery mode (median C5a level 51.4 vs. $56.2 \mathrm{ng} / \mathrm{ml} ; p=0.748$ and median RANTES level 207.6 vs. $171.6 \mathrm{ng} / \mathrm{ml} ; p=0.402$, for vaginal and non-vaginal delivery, respectively).

Correlation analysis revealed a significant positive correlation between serum levels of C5a and CRP (Spearman $\left.r^{2}=0.33 ; p=0.010\right)$.

\section{Discussion}

The early identification of neonates with EONS is critical, because it allows clinicians to implement suitable and more effective medical intervention improving the outcome. Reliable ruling-out of sepsis is also important to avoid exposure to broad-spectrum antibiotics (which, even if for only a few days, might foster antibiotics resistance patterns) and the long-term effects of antibiotic overuse in patients. The lack of a reliable test for early diagnosis of EONS forces a search for new biomarkers. In this preliminary study, 3 novel markers such as C5a, IL-17A and RANTES were measured in neonates with EONS and non-infected newborns for their potential use in the diagnosis of early-onset neonatal sepsis.

In the current preliminary study, for the first time significantly higher levels of C5a were found in neonates with 

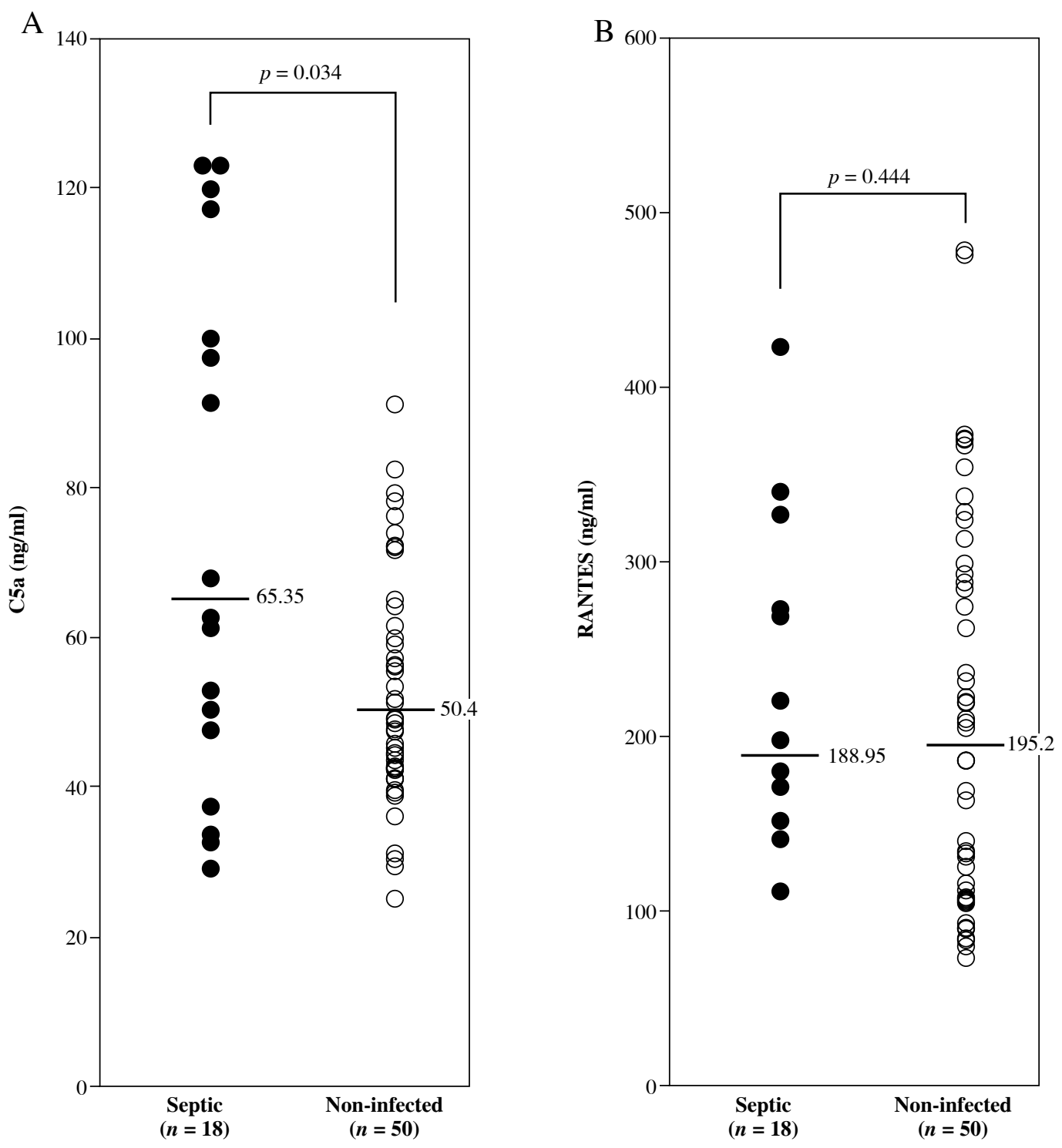

Fig. 1. Scatter plots representing C5a (A) and RANTES (B) levels in serum of neonates. The C5a levels measured in septic neonates (EONS) vs. non-infected neonates (healthy controls) showed a significant difference $(p=0.034$; by Mann-Whitney U test)

EONS compared with non-infected cases. These results are in accordance with the recent study by Unnewehr et al. who showed a significant increase of C5a concentrations in adult patients during septic shock [17]. Taken together, these results and data from experimental models linking elevated C5a with immunoparalysis [24], multi-organ failure [25], imbalances in the coagulation system [26] and the apoptosis of thymocytes [27], suggest that C5a increases in early phases of infection and may be an important factor for the inflammatory process in the pathogenesis of sepsis.

Regarding IL-17A, significantly increased production of this interleukin was observed in two previous studies conducted in adult septic patients [10,11], while little is known about IL-17A levels in neonates, especially those with EONS. Contrary to the findings in adults, in the current study IL-17A concentrations were under the detected level in both EONS and non-infected cases. Nevertheless, the results are in accordance with the previous study by Papoff et al. comparing IL-17 expression in body fluids from 13 sick and 7 healthy neonates admitted to NICU [13]. Undetectable levels of IL-17 were found in all plasma samples of infected neonates as well as those with inflammatory non-infectious diseases and controls. This is in line with a recent study by Caron et al., who examined neonatal 
production of IL-17 in mixed mononuclear cells (MMCs) isolated from umbilical cord blood and found severely depressed IL-17 production by neonatal MMCs compared to adults [12]. In addition, reduced intracellular formation of IL-17, and diminished IL-17 response to both group B streptococci (GBS) and Escherichia coli, were observed [12]. Taking into account the fact that in healthy children serum levels of IL-17 increase with age [28], low or undetectable IL-17 levels observed in neonates may mirror the immaturity of the neonatal immune system to launch an effective Th17 response.

Most of the previous studies showed lower RANTES concentration in septic neonates compared with healthy subjects [19-22], though diametrically opposite results have also been reported in the setting of EONS [23]. In the current study the median concentration of this chemokine was lower in EONS group then in non-infected neonates, but the difference did not reach statistical significance. This discrepancy in the results between the current and previous studies may be attributed to the relatively small sample size, differences in timing of evaluation (early vs. late onset-sepsis) or varied patient population (including term vs. premature neonates). It must be also underlined, that at the time of sepsis onset, none of the septic neonates enrolled into the current study had significant thrombocytopenia and because platelets are principal reservoir of RANTES in peripheral circulation [29], this may account for the lack of significant difference in chemokine concentration between the groups.

This study has some limitations which need to be pointed out. Firstly, none of the included neonates had a positive blood culture. Low and/or intermittent bacteraemia or small blood inoculation volumes may account for this. Confirmation of this fact are data obtained in the ESGNI-001 study in which the percentage of positive microbiological blood culture among patients with symptoms of bloodstream infections was $15 \%$ in European countries [31]. These results arouse mistrust in clinicians to this diagnostic test. Secondly, due to ethical reasons the marker evaluations were performed in a single serum sample collected within the first 72 hours of life. Serial measurements of the parameters could be more informative, especially when considering their use for the prognosis or treatment monitoring.

In conclusion, the results of our study obtained in this small number of patients are very preliminary nonetheless showed that C5a is a very interesting biomarker. Thus, there is a strong need for further studies evaluating the dynamics of C5a and its sensitivity and specificity in the diagnosis of EONS on a large number of neonates. Moreover, it is also necessary to differentiate the usefulness of C5a determination between EONS and other pathological conditions, such as necrotizing enterocolitis, meconium aspiration syndrome, hypoxia and respiratory failure of varying etiology. We conclude that such studies will help to assess whether this anaphylatoxin could be useful laboratory tool for differential diagnosis of infectious vs. non-infectious systemic inflammatory response.

This work was supported by the Polish National Science Centre (Grant no. N N407 115340). The authors acknowledge Bożenna Wołowska for outstanding laboratory assistance.

The authors declare no conflict of interest.

\section{References}

1. Polin RA, Comm Fetus N (2012): Management of Neonates With Suspected or Proven Early Onset Bacterial Sepsis. Pediatrics 129: 1006-1015.

2. Chiesa C, Panero A, Osborn JF, et al. (2004): Diagnosis of neonatal sepsis: A clinical and laboratory challenge. Clin Chem 50: 279-287.

3. Pammi M, Flores A, Leeflang M, Versalovic J (2011): Molecular Assays in the Diagnosis of Neonatal Sepsis: A Systematic Review and Meta-analysis. Pediatrics 128: E973-E985.

4. Prucha M, Bellingan G, Zazula R (2015): Sepsis biomarkers. Clin Chim Acta 440: 97-103.

5. Machado JR, Soave DF, da Silva MV, et al. (2014): Neonatal Sepsis and Inflammatory Mediators. Mediators Inflamm 2014: 1-10.

6. Delanghe J, Speeckaert M (2015): Translational research and biomarkers in neonatal sepsis. Clin Chim Acta 451: 46-64.

7. Rittirsch D, Flierl MA, Ward PA (2008): Harmful molecular mechanisms in sepsis. Nat Rev Immunol 8: 776-787.

8. Weaver CT, Hatton RD, Mangan PR, Harrington LE (2007): IL-17 family cytokines and the expanding diversity of effector T cell lineages. Annu Rev Immunol 25: 821-852.

9. Flierl MA, Rittirsch D, Gao H, et al. (2008): Adverse functions of IL-17A in experimental sepsis. FASEB J 22: 21982205 .

10. Li J, Li M, Su LX, et al. (2015): Alterations of T helper lymphocyte subpopulations in sepsis, severe sepsis, and septic shock: a prospective observational study. Inflammation 38: 995-1002.

11. Wu HP, Shih CC, Chu CM, et al. (2015): Effect of interleukin-17 on in vitro cytokine production in healthy controls and patients with severe sepsis. J Formos Med Assoc 114: 12501257.

12. Caron JE, La Pine TR, Augustine NH, et al. (2014): Severely depressed interleukin-17 production by human neonatal mononuclear cells. Pediatr Res 76: 522-527.

13. Papoff P, Fiorucci P, Lenti L, et al. (1999): Characterization of interleukin (IL)-17 expression in the human neonate. Pediatr Res 45: 900-900.

14. Rittirsch D, Flierl MA, Nadeau BA, et al. (2008): Functional roles for C5a receptors in sepsis. Nat Med 14: 551-557.

15. Ward PA (2004): The dark side of C5A in sepsis. Nat Rev Immunol 4: 133-142.

16. Czermak BJ, Sarma V, Pierson CL, et al. (1999): Protective effects of C5a blockade in sepsis. Nat Med 5: 788-792.

17. Unnewehr H, Rittirsch D, Sarma JV, et al. (2013): Changes and Regulation of the C5a Receptor on Neutrophils during Septic Shock in Humans. J Immunol 190: 4215-4225. 
18. Cavaillon JM, Adib-Conquy M, Fitting C, et al. (2003): Cytokine cascade in sepsis. Scand J Infect Dis 35: 535-544.

19. Ng PC, Li K, Leung TF, et al. (2006): Early prediction of sepsis-induced disseminated intravascular coagulation with interleukin-10, interleukin-6, and RANTES in preterm infants. Clin Chem 52: 1181-1189.

20. Sugitharini V, Prema A, Thangam EB (2013): Inflammatory mediators of systemic inflammation in neonatal sepsis. Inflamm Res 62: 1025-1034.

21. Manoura A, Gourgiotis D, Galanakis E, et al. (2010): Circulating concentrations of alpha- and beta-chemokines in neonatal sepsis. Int J Infect Dis 14: E806-E809.

22. Shouman B, Badr R (2010): Regulated on activation, normal $\mathrm{T}$ cell expressed and secreted and tumor necrosis factor-alpha in septic neonates. J Perinatol 30: 192-196.

23. Wasek-Buko M, Behrendt J, Stojewska M, et al. (2007): P1546 Serum chemokin RANTES as a immunological indicator of early-onset neonatal sepsis. Int J Antimicrob Agents 29: $\mathrm{S} 433$.

24. Huber-Lang MS, Younkin EM, Sarma JV, et al. (2002): Complement-induced impairment of innate immunity during sepsis. J Immunol 169: 3223-3231.

25. Huber-Lang M, Sarma VJ, Lu KT, et al. (2001): Role of C5a in multiorgan failure during sepsis. J Immunol 166: 11931199.

26. Laudes IJ, Chu JC, Sikranth S, et al. (2002): Anti-C5a ameliorates coagulation/fibrinolytic protein changes in a rat model of sepsis. Am J Pathol 160: 1867-1875.

27. Riedemann NC, Guo RF, Laudes IJ, et al. (2002): C5a receptor and thymocyte apoptosis in sepsis. FASEB J 16: 887-888.

28. Kleiner G, Marcuzzi A, Zanin V, et al. (2013): Cytokine Levels in the Serum of Healthy Subjects. Mediators Inflamm 2013: 1-6.

29. Appay V, Rowland-Jones SL (2001): RANTES: a versatile and controversial chemokine. Trends Immunol 22: 83-87.

30. Olsen IE, Groveman SA, Lawson ML, et al. (2010): New Intrauterine Growth Curves Based on United States Data. Pediatrics 125: E214-E24.

31. Bouza E, Perez-Molina, Muńoz P, et al. (1999): Report of ESGNI-001 and ESGNI-002 studies. Bloodstream infections in Europe. Clin Microbiol Infect 5: 2S1-2S12. 